Pacific Journal of Mathematics

GEL'FAND THEORY IN ALGEBRAS OF DIFFERENTIABLE 


\title{
GELFAND THEORY IN ALGEBRAS OF DIFFERENTIABLE FUNCTIONS ON BANACH SPACES
}

\author{
José E. GaLE \\ Dedicated to J. M. Ortega
}

\begin{abstract}
We study algebras of differentiable functions on a reflexive Banach space, defined by polynomial approximation on bounded sets. We find the spectra of such algebras and we investigate the structure of their closed ideals. In relation to this, we treat also an approximation problem of functions $f$ such that $f, f^{1}, \ldots, f^{(m)}$ vanish on a weakly compact set by a method involving radical algebras and the Ahlfors-Heins theorem.
\end{abstract}

Introduction. We denote by $E$ a complex reflexive Banach space with closed unit ball $\Omega$, by $E^{\prime}$ its topological dual, and by $m$ a fixed non-negative integer. If $S$ is a subset of $E$ and $Y$ a Banach space, a function $g: S \rightarrow Y$ is said to be weakly continuous if it is continuous with respect to the weak topology on $S$ and the norm topology on $Y$. We denote by $C_{w b}^{m}(\Omega)$ the algebra of the $m$ times continuously differentiable complex valued functions whose $m$ derivatives can be extended by continuity to the boundary of $\Omega$ and, moreover, these derivatives are weakly continuous on $\Omega$. Let $\tau^{m}$ be the topology on $C_{w b}^{m}(\Omega)$ of $m$-uniform convergence on $\Omega$. Equipped with $\tau^{m}, C_{w b}^{m}(\Omega)$ becomes a Banach algebra.

We are interested in functions obtained by polynomial approximation. For this, we consider $A^{m}(\Omega)$, defined as the $\tau^{m}$-completion of $P_{f}$ in $C_{w b}^{m}(\Omega)$. Here $P_{f}$ denotes the algebra of the polynomials of finite type on $E$, i.e. the ones generated by the elements of $E^{\prime}$ and their conjugates, and the constant functions; it is clear that $P_{f} \subset C_{w b}^{m}(\Omega)$. We may define also the spaces $A^{\infty}(\Omega), A^{m}(E), A^{\infty}(E)$ in a similar manner to $A^{m}(\Omega)$ (definitions below). In fact, the functions of type of $A^{m}(\Omega)$ have been introduced in the literature in relation with approximation questions ([1], [2], [16]). For instance, when $E^{\prime}$ has the bounded approximation property, $A^{m}(\Omega)=$ $C_{w b}^{m}(\Omega)$ for every $m$ (see [2]). Also, the weak continuity has been treated recently in great detail (see [3]), for instance). Here we study the spaces $A^{m}(\Omega), A^{\infty}(\Omega), A^{m}(E), A^{\infty}(E)$ as topological algebras.

In $\S 1$ the Gelfand's theory in the Banach algebra $A^{m}(\Omega)$ is given: we show that $\Omega$ is the spectrum of $A^{m}(\Omega)$ and the identification between the 
Gelfand topology and weak topology on $\Omega$ is obtained (we call spectrum of a topological algebra the set of its non-zero complex continuous homomorphisms). Also, $A^{m}(\Omega)$ is a regular algebra. We begin the study of the structure of the closed ideals in $A^{m}(\Omega)$. The minimal primary closed ideals are characterized and it allows us to prove-in $\$ 2$-in this context a generalization of a Borel's classical result about formal series. The spectral synthesis problem is raised and some partial results are given.

In $\S 2$ we discuss the case of the algebras $A^{\infty}(\Omega), A^{m}(E), A^{\infty}(E)$.

We study in $\$ 3$ a problem of approximation. Let $f \in A^{m}(\Omega)$ such that $f, D f, \ldots, D^{m} f$ vanish on a weakly compact subset $K$ of $\Omega$. Does there exist a sequence $\left\{f_{n}\right\}_{n=1}^{\infty}$ of elements of $A^{m}(\Omega)$ vanishing over a weak neighborhood of $K$ (depending on $n$ ) such that $\lim _{n \rightarrow \infty} f_{n}=f$ for $\tau^{m}$ in $A^{m}(\Omega)$ ? This question is obviously related with spectral synthesis. We do not solve completely this problem, but we obtain some consistent partial results. The method consists in considering the map $t \mapsto f^{t}(\operatorname{Re} t>m)$ in case when $f \geq 0$. We obtain an analytic map from the half-plane $\{t \in C \mid \operatorname{Re} t$ $>m$ \} into $A^{m}(\Omega)$ which clearly defines a semigroup and has exponential growth on vertical lines. Using the Ahlfors-Heins theorem as in [7] we see that $f^{t} \in \operatorname{Ker} \varphi$ for $\operatorname{Re} t>m$ if $\varphi$ is any continuous homomorphism from $\left\{g \in A^{m}(\Omega) \mid g(K)=0\right\}$ into a radical algebra. For $m=1$, this shows in particular that the answer to the approximation problem is positive in the case where $f \geq 0$ or $f^{-1}(0)=K(f$ real-valued $)$.

Sections 1 and 2 of this paper are parts of the author's doctoral thesis (Zaragoza, Spain, 1980). The content of $\S 3$ was obtained in collaboration with Jean Esterle during the stay of the author in the Universite de Bordeaux I (France), the academic year 1981-82, supported by a Beca del Plan de Formación de Personal Investigador del M.E.C., Spain.

1. Gelfand theory in $A^{m}(\Omega)$. The space $C_{w b}^{m}(\Omega)$ is a Banach algebra with the norm defined by $\|f\|_{m}=\sup _{x \in \Omega} \sum_{j=0}^{m}\left\|D^{j} f(x)\right\|\left(f \in C_{w b}^{m}(\Omega)\right)$, and $A^{m}(\Omega)$ is a Banach subalgebra. We observe that $\psi \circ f \in A^{m}(\Omega)$ if $f \in A^{m}(\Omega)$ and $\psi$ is any $m$ times continuously differentiable function $\psi$ : $U \rightarrow C$, with $U$ some open subset of $C$, such that $f(\Omega) \subset U$. It is a consequence of the polynomial approximation and routine calculations on the formulae for high derivatives ([8], p. 161). So, if $f \in A^{m}(\Omega)$ and $f(x) \neq 0$ for all $x \in \Omega$, the function $(1 / f)(x)=1 / f(x),(x \in \Omega)$ is in $A^{m}(\Omega)$ (it suffices to consider $1 / f=\alpha \circ f$, with $\alpha(z)=z^{-1}$, if $z \in C \backslash\{0\}$ ).

Proposition (1.1). Let $E$ be a reflexive Banach space. Then $\Omega$ is the spectrum of the Banach algebra $A^{m}(\Omega)$ and the Gelfand topology equals the weak topology on $\Omega$. 
Proof. According to the general rule of [9], p. 22, 23, the spectrum of $A^{m}(\Omega)$ is $\Omega$. Moreover, $\Omega$ is a weakly compact subset of $E$ and the functions in $A^{m}(\Omega)$ are weakly continuous on $\Omega$. Thus, in accordance with [9], p. 40 , Th. $1^{\prime}$, the Gelfand topology equals the weak topology on $\Omega$.

Proposition (1.2). If $E$ is a reflexive Banach space, $A^{m}(\Omega)$ is a regular algebra.

Proof. If $x_{0} \in \Omega$ and $S$ is a Gelfand-closed subset of $\Omega$ with $x_{0} \notin S$ we may assume that $S=\{x \in \Omega:|g(x)| \geq 1\}$ for some $g \in A^{m}(\Omega)$ such that $g\left(x_{0}\right)=0$. Therefore, by the observation above to proposition (1.1), it suffices to take $\psi$ verifying $\psi(0)=1$ and $\psi(z)=0$ for $|z| \geq 1$, and consider $\psi \circ g$.

The Shilov's theory of ideals shows that for any Gelfand-closed $S \subset \Omega$ there exists an ideal $J(S)$ in $A^{m}(\Omega)$, minimal among all ideals $I$ satisfying $Z(I)=S(Z(I)$ denotes the zero set of $I)$. The ideal $J(S)$ is formed by the functions $g \in A^{m}(\Omega)$ vanishing on some Gelfand-neighborhood of $S$ in $\Omega$. If $S=\left\{x_{0}\right\}, x_{0} \in \Omega, \overline{J\left(x_{0}\right)}$ is the minimal primary closed ideal corresponding to $x_{0}$. We are going characterize $\overline{J\left(x_{0}\right)}$ by means of the derivatives of its elements. Henceforth in this section $E$ denotes a separable Hilbert space with a fixed orthonormal basis $\left\{e_{n}\right\}_{n=1}^{\infty}$ and $\left\{P_{n}\right\}_{n=1}^{\infty}$ designating the sequence of projections associated to this basis.

LEMMA (1.3). Let $K$ be a weakly closed subset of $\Omega$. Let $f \in A^{m}(\Omega)$ such that $D^{r} f(K)=0$ for every $r=0,1, \ldots, m$. Then, for $\varepsilon>0$ given there exists $\rho>0$ such that $\left\|D^{r} f(x)\right\|<\varepsilon d(x, K)^{m-r}$ whenever $d(x, K)<\rho,(x \in \Omega)$. $(d(x, K)$ denotes the distance in norm from $x$ to $K$.)

Proof. The derivative $D^{m} f: \Omega \rightarrow L^{m}(E ; C), L^{m}(E ; C)$ being the space of continuous $m$-linear mappings on $E$, is weakly continuous and $\Omega$ is weakly compact, so $D^{m} f$ is uniformly weakly continuous and, moreover, uniformly continuous (in norm). Therefore we can find $\rho>0$ such that $d(x, K)<\rho,(x \in \Omega)$ implies $\left\|D^{m} f(x)\right\|<\varepsilon$. Now, if $y \in K$,

$$
\begin{aligned}
D^{m-1} f(x) & =D^{m-1} f(x)-D^{m-1} f(y) \\
& =\int_{0}^{1} D^{m} f(y+t(x-y))(x-y) d t .
\end{aligned}
$$

We have

$$
\left\|D^{m-1} f(x)\right\| \leq \sup _{t \in[0,1]}\left\|D^{m} f(y+t(x-y))\right\|\|x-y\|
$$


and $\left\|D^{m-1} f(x)\right\|<\varepsilon d(x, K)$ is easily obtained. By repeating this process, we have $\left\|D^{r} f(x)\right\|<\varepsilon d(x, K)^{m-r}(r=0,1, \ldots, m)$.

We note that, if $f \in A^{m}(\Omega)$, then $\lim _{n \rightarrow \infty} f \circ P_{n}=f$ in $A^{m}(\Omega)$. (See [16]. The proof is similar for $m>1$ and functions defined on $\Omega$.)

ThEOREM (1.4). If $x_{0} \in \Omega$ and $I\left(x_{0}\right)=\left\{f \in A^{m}(\Omega): D^{r} f\left(x_{0}\right)=0\right.$ $(r=0,1, \ldots, m)\}$, then $I\left(x_{0}\right)=\overline{J\left(x_{0}\right)}$.

Proof. We choose an infinitely differentiable function $h$ on $E$ such that $h(x)=1$, for $\|x\|<\xi$ and $h(x)=0$ for $\|x\| \geq c ; \xi, c$ being positive real numbers. For every $n \in N$, we put $h_{n}=h \circ P_{n}, f_{n}=f \circ P_{n}$, where $f \in A^{m}(\Omega)$, and if $x \in E, n, \nu \in N, h_{n, \nu}(x)=h_{n}\left(\nu\left(x-x_{0}\right)\right)$. We have $h_{n, \nu}(x)=1$ if and only if $\left\|P_{n} x-P_{n} x_{0}\right\|<\xi / \nu, h_{n, \nu}(x)=0$ iff $\| P_{n} x-$ $P_{n} x_{0} \| \geq c / \nu$. In this last case, $D^{r} h_{n, \nu}(x)=0(r=0,1, \ldots, m)$.

We choose $n(\nu)$ such that $\left\|P_{n(\nu)} \mathrm{x}_{0}-\mathrm{x}_{0}\right\|<c / \nu$, for each $\nu \in N$. If $f \in I\left(x_{0}\right)$, there exists $\rho>0$ such that $\left\|x-x_{0}\right\|<\rho$ implies

$$
\left\|D^{r} f(x)\right\|<\varepsilon\left\|x-x_{0}\right\|^{m-r} \quad(r=0,1, \ldots, m) .
$$

We take $\nu$ such that $2 c / \nu<\rho$. Then, if

$$
\left\|P_{n(\nu)} x-P_{n(\nu)} x_{0}\right\| \geq c / \nu, \quad D^{k} h_{n(\nu), \nu}(x)=0(k=0,1, \ldots, m)
$$

and if $\left\|P_{n(\nu)} x-P_{n(\nu)} x_{0}\right\|<c / \nu$,

$$
\left\|P_{n(\nu)} x-x_{0}\right\| \leq\left\|P_{n(\nu)} x-P_{n(\nu)} x_{0}\right\|+\left\|P_{n(\nu)} x_{0}-x_{0}\right\|<\frac{2 c}{\nu},
$$

and so

$$
\begin{aligned}
\left\|D^{r-k} f_{n(\nu)}(x)\right\| & =\left\|D^{r-k} f\left(P_{n(\nu)} x\right)\right\| \\
& <\varepsilon\left(\frac{2 c}{\nu}\right)^{m n-r+k} \quad(r=0,1, \ldots, m ; k=0,1, \ldots, r) .
\end{aligned}
$$

It follows that, for every $x \in \Omega$,

$$
\begin{aligned}
\left\|D^{r}\left(h_{n(\nu), \nu} f_{n(\nu)}\right)(x)\right\| & \leq \sum_{k=0}^{r}\left(\begin{array}{l}
r \\
k
\end{array}\right)\left\|D^{k} h_{n(\nu), \nu}(x)\right\|\left\|D^{r-k} f_{n(\nu)}(x)\right\| \\
& \leq \sum_{k=0}^{r}\left(\begin{array}{l}
r \\
k
\end{array}\right) \nu^{k}\|h\|_{m} \varepsilon\left(\frac{2 c}{\nu}\right)^{m-r+k}=c^{\prime}\|h\|_{m} \varepsilon,
\end{aligned}
$$

where $c^{\prime}$ is a constant number.

We have proved that $\lim _{\nu \rightarrow \infty} h_{n(\nu), \nu} f_{n(\nu)}=0$ in $A^{m}(\Omega)$. But

$$
\left(1-h_{n(\nu), \nu}\right) f_{n(\nu)} \in J\left(x_{0}\right),
$$

because the sets $\left\{\underline{x \in \Omega}:\left\|P_{n} x-P_{n} x_{0}\right\|<\eta\right\}_{\eta>0, n \in N}$ are Gelfand-neighborhoods. So, $f \in \overline{J\left(x_{0}\right)}$. 
The problem of spectral synthesis for $A^{m}(\Omega)$ consists in knowing if each closed ideal $I \subset A^{m}(\Omega)$ is the intersection of primary closed ideals which contain it. An equivalent formulation is to pose if the equality

$$
I=\bigcap_{x \in Z(I)} \overline{I+I(x)}
$$

is true. When $E$ is finite-dimensional the equality is satisfied (Whitney theorem; see [19], p. 89, for instance). Indeed, the Whitney theorem in finite dimensions states that $\bar{I}=\bigcap_{x \in Z(I)} I+I(x)$, for every ideal $I$ of $A^{m}(\Omega)$. The following counterexample of [11], p. 173, shows that this last equality is not verified in Hilbert space case: we consider $I$ as the ideal generated in $A^{1}(\Omega)$ by the functions $\left\{g_{n}: n=1,2, \ldots\right\}$ defined as $g_{n}(x)=$ $\left\langle x \mid e_{n}\right\rangle(x \in E)$, where $\langle\mid\rangle$ is the inner product in $E$. We have $\overline{I+I(0)}$ $=\left\{f \in A^{1}(\Omega): f(0)=0\right\}$ whereas $I+J(0) \neq\left\{f \in A^{1}(\Omega): f(0)=0\right\}$. In general, we observe that $(*)$ is true when $I=I\left(x_{0}\right)$, for some $x_{0} \in \Omega$. Let $K$ be a weakly closed subset of $\Omega$. We define

$$
I_{p}(K)=\left\{f \in A^{m}(\Omega): D^{r} f(K)=0(r=0,1, \ldots, p)\right\} .
$$

If $\overline{J(K)}=I_{m}(K)$, then $\overline{J(K)}=\bigcap_{x \in K} I(x)$ and $(*)$ is also true for $\overline{J(K)}$. On the other hand, if $\overline{J(K)} \neq I_{m}(K)$ the spectral synthesis is not possible in $A^{m}(\Omega)$. Thus, to know if any $m$-null function on a weakly compact subset of $\Omega$ can be approximated in $A^{m}(\Omega)$ by functions vanishing on some weak neighborhood of the weakly compact is an approximation problem, previous to synthesis problem. The proof of $\overline{J(K)}=I_{m}(K)$ (and also the one of synthesis), in the case finite-dimensional depends substantially on the dimension and we have not been able to find a proof of a different nature, valid for any weakly compact subset of $\Omega$. Nevertheless, certain partial results can be stated. For example, when $K$ is such that $P_{n}(K) \subset K$ for $n$ sufficiently large, $I_{m}(K)=\overline{J(K)}$ is obtained, by reducing the question to finite dimensional case. Another type of result can be proved by using the ideas of Theorem (1.4).

Proposition (1.5). Let $K$ be a convex norm-compact in $\Omega$. Then $I_{1}(K)=\overline{J(K)}$ in $A^{1}(\Omega)$.

Proof. Let $h$ be a function in $C^{\infty}\left(R^{n}\right)$ such that $h(a)=1$ for $\|a\|<\xi$, $h(a)=0$ for $\|a\| \geq c, a \in R, \xi, c>0$ fixed. We define $h_{n, v}(x)=$ $h\left(\nu d\left(P_{n} x, P_{n}(K)\right)\right)$, for every $x \in \Omega$, where $d\left(P_{n} x, P_{n}(K)\right)=$ $\inf _{y \in P_{n}(K)}\left\|P_{n} x-y\right\|$. This last function is differentiable in $P_{n}(E) \backslash P_{n}(K)$ and, $P_{n}(K)$ being closed convex, there is a continuous mapping $\pi_{n}$ such 
that $d\left(x_{n}, P_{n}(K)\right)=\left\|x_{n}-\pi_{n}\left(x_{n}\right)\right\|$, for all $x_{n} \in P_{n}(E)$. Also, $\operatorname{Dd}\left(\cdot, P_{n}(K)\right)_{x_{n}}=D\|\|_{x_{n}-\pi_{n}\left(x_{n}\right)},\|\|$ denoting the euclidean norm in $P_{n}(E)$ (see [21], p. 140).

We choose $n(\nu)$ such that $\sup _{y \in K}\left\|P_{n(\nu)} y-y\right\|<c / \nu$ for each $\nu \in N$. If $d\left(P_{n(\nu)} x, P_{n(\nu)}(K)\right)<c / \nu$, then

$$
\begin{gathered}
\left\|D h_{n(\nu), \nu}(x)\right\|=\nu\left\|D h\left(\nu d\left(P_{n(\nu)} x, P_{n(\nu)}(K)\right)\right)\right\|, \\
\|D\|\left\|_{P_{n(\nu)} x-\pi_{n(\nu)}\left(P_{n(\nu)} x\right)}\right\| \leq \nu\|h\|_{1} .
\end{gathered}
$$

Let $\varepsilon>0$. If $f \in I_{1}(K)$ there exists $\rho>0$ such that $d(x, K)<\rho$ implies $\left\|D^{r} f(x)\right\| \leq \varepsilon d(x, K)^{1-r}(r=0,1)$, according to Lemma (1.3). Therefore, choosiing $\nu$ to have $2 c / \nu<\rho$, we obtain

$$
\begin{aligned}
\left\|D^{r} h_{n(\nu), \nu}\left(f \circ P_{n(\nu)}\right)(x)\right\| & \leq \sum_{j=0}^{r}\left\|D^{j} h_{n(\nu), \nu}(x)\right\|\left\|D^{r-j}\left(f \circ P_{n(\nu)}\right)(x)\right\| \\
& \leq \sum_{j=0}^{r}\|h\|_{1} \nu^{j} \frac{(2 c)^{1-r+j}}{\nu^{1-r+j}} \varepsilon,
\end{aligned}
$$

for every $x \in \Omega(r=0,1)$. So

$$
\lim _{\nu \rightarrow \infty} h_{n(\nu), \nu}\left(f \circ P_{n(\nu)}\right)=0 \quad \text { in } A^{1}(\Omega) \quad \text { and } \quad f \in \overline{J(K)} .
$$

2. Gelfand theory in the algebras $A^{\infty}(\Omega), A^{m}(E), A^{\infty}(E)$. The space $C_{w b}^{\infty}(\Omega)=\bigcap_{n=0}^{\infty} C_{w b}^{n}(\Omega)$ equipped with the topology $\tau^{\infty}$ of uniform convergence on $\Omega$ of the functions and their derivatives is a Fréchet algebra. We are also interested in functions defined on all of $E$. Thus, we consider $C_{w b}^{m}(E)$, the space formed by the $m$ times continuously differentiable functions on $E$ whose $m$ derivatives are weakly continuous on the bounded subsets of $E$. With the topology $\tau_{b}^{m}$ of $m$-uniform convergence on the bounded subsets of $E, C_{w b}^{m}(E)$ is also a Fréchet algebra. Finally, if $C_{w b}^{\infty}(E)=\bigcap_{n=0}^{\infty} C_{w b}^{n}(E)$ we define obviously the topology $\tau_{b}^{\infty}$ on $C_{w b}^{\infty}(E)$ and $C_{w b}^{\infty}(E)$ is again a Fréchet algebra. Now, we define $A^{\infty}(\Omega), A^{m}(E)$, $A^{\infty}(E)$ as the Fréchet subalgebras obtained by completing $P_{f}$ in the respective $C_{w b}^{\infty}(\Omega), C_{w b}^{m}(E), C_{w b}^{\infty}(E)$.

In the following proposition we denote by $\tau_{G}^{q}(0 \leq q \leq \infty)$ the Gelfand topology on $E$ defined by $A^{q}(E)$, and by $\tau$ the topology on $E$ defined by " $S \subset E$ is $\tau$-closed iff $S \cap \Omega_{p}$ is weakly closed in $\Omega_{p}=\{x \in E:\|x\| \leq p\}$, for every $p>0$ ".

Proposition (2.1) Let $E$ be a reflexive Banach space. Then, (i) $\operatorname{Spec} A^{\infty}(\Omega)=\Omega$ and the Gelfand topology equals the weak topology on $\Omega$. 
(ii) $\operatorname{Spec} A^{m}(E)=E$, $\operatorname{Spec} A^{\infty}(E)=E$ and if $m_{1}, m_{2} \in N$ with $m_{1} \leq m_{2}$, then

$$
\sigma\left(E, E^{\prime}\right)<\tau_{G}^{\infty}<\tau_{G}^{m_{2}}<\tau_{G}^{m_{1}}<\tau_{G}^{0}=\tau<\beta\left(E, E^{\prime}\right)
$$

Proof. (i) Each $x \in \Omega$ defines a $\varphi_{x} \in \operatorname{Spec} A^{\infty}(\Omega)$ as $\varphi_{x}(f)=f(x)$, for every $f \in A^{\infty}(\Omega)$. Conversely, if $\varphi \in \operatorname{Spec} A^{\infty}(\Omega)$ there exists $n \in N \cup\{0\}$ such that $\varphi$ is a $\tau^{n}$-continuous homomorphism on $A^{\infty}(\Omega)$ and, $A^{\infty}(\Omega)$ being dense in $A^{n}(\Omega)$, there is a $\psi \in \operatorname{Spec} A^{n}(\Omega), \tau^{n}$-continuous extension of $\varphi$. According to the proposition (1.2) it follows that, for some $x_{0} \in \Omega$, $\varphi(g)=\psi(g)=g\left(x_{0}\right)$ whenever $g \in A^{\infty}(\Omega)$. Moreover, the Gelfand and weak topologies on $\Omega$ are equal since the functions of $A^{\infty}(\Omega)$ are weakly continuous on $\Omega$ and, on the other hand, $E^{\prime} \subset A^{\infty}(\Omega)$.

(ii) The algebra $A^{m}(E)$ is the projective limit of the system formed by the Banach algebras $A^{m}\left(\Omega_{p}\right)(p=1,2, \ldots)$ together with the natural restriction mappings. Therefore, it follows that the mapping $\Gamma: x \in \lim _{\rightarrow} \Omega_{p}$ $\cong E \mapsto \Gamma(x) \in \operatorname{Spec} A^{m}(E)$ such that $\Gamma(x)(f)=f(x) \quad(x \in E, \vec{f} \in$ $\left.A^{m}(E)\right)$, is bijective and continuous $\left([12]\right.$, p. 160). The case $A^{\infty}(E)$ is similar.

Now, the topology $\tau$ defined as above is the one of $E$ considered as $\lim _{\rightarrow} \Omega_{p}$, with the weak topology in each $\Omega_{p}$. If $C\left(E_{\tau}\right)=\{g: E \rightarrow C: g$ $\tau$-continuous $\}$ and $f \in A^{0}(E)$, then $f \cong f \circ \Gamma \in C\left(E_{\tau}\right)$. Conversely, if $f \in$ $C\left(E_{\tau}\right), f$ is weakly continuous on each $\Omega_{p}(p \in N)$. So, $f \in A^{0}(E)$ ([2], p. 203), and $C\left(E_{\tau}\right)=A^{0}(E)$. Finally, $\tau$ is compatible with the vectorial structure of $E$ ([18], p. 151), whence $E_{\tau}$ is a completely regular topological space; in particular, $\tau$ equals the topology on $E$ defined by the functions of $C\left(E_{\tau}\right)$. In short, $\tau_{G}^{0}=\tau$.

The inequalities between the several topologies of the statement are evident.

REMARK. (i) We do not know whether the topologies $\tau_{G}^{\infty}, \tau_{G}^{n}, \tau_{G}^{0}, n \in N$, are identical or distinct on $E$. Nevertheless, it is immediate that $\tau_{G}^{0} \neq$ $\beta\left(E, E^{\prime}\right)$ (there are $\beta\left(E, E^{\prime}\right)$-continuous functions on $E$ which are not in $A^{0}(E)$ ) and $\sigma\left(E, E^{\prime}\right) \neq \tau_{G}^{\infty}:$ if $E$ is infinite dimensional, then there is always a sequence of norm one linear functionals $\left(\phi_{n}\right)$ on $E$ such that $\phi_{n} \rightarrow 0$ for the $*$-weak topology ([15]). Thus, the function $\sum_{n=1}^{\infty} \phi_{n}^{n} / 2^{n}$ is not weakly continuous on $E$ but is in $A^{\infty}(E)$, and $\sigma\left(E, E^{\prime}\right)<\tau_{G}^{\infty}$.

The same reasoning as above in $\S 1$ shows that $A^{\infty}(\Omega), A^{m}(E), A^{\infty}(E)$ are regular algebras. Henceforth $E$ denotes a separable Hilbert space with $\left\{e_{n}\right\}_{n=1}^{\infty},\left\{P_{n}\right\}_{n=1}^{\infty}$ as indicated in Lemma (1.3). A result as Theorem (1.4) 
can be obtained for the algebras $A^{\infty}(\Omega), A^{m}(E), A^{\infty}(E)$. Moreover, if $E=R^{p}$ a classical result of $E$. Borel identifies the algebra $A^{\infty}\left(R^{p}\right) / I(x)$, $x \in R^{p}$, as the algebra formed by all formal power series in $p$ variables. We shall generalize this proposition in our context, by means of Theorem (1.4) and a known theorem about surjectivity between Fréchet spaces. Let $\mathscr{S}_{w b}(E)=\prod_{m=0}^{\infty} P_{w b}\left({ }^{m} E\right), P_{w b}\left({ }^{m} E\right)$ being the set of the $m$-homogeneous polynomials which are weakly continuous on the balls of $E$. With the usual operations and the topology determined by the seminorns family $\sigma_{n}(\beta)=\sup _{0 \leq k \leq n}\left\|\beta_{k}\right\|$, for every $\beta=\left(\beta_{0}, \ldots, \beta_{n}, \ldots\right) \in \mathscr{S}_{w b}(E), \mathscr{S}_{w b}(E)$ is a Fréchet algebra.

TheOREM (2.3). Let $x_{0} \in E$ be. If $\beta=\left(\beta_{0}, \ldots, \beta_{n}, \ldots\right) \in \mathscr{S}_{w b}(E)$ there exists $f \in A^{\infty}(E)$ such that $\hat{d}_{n} f\left(x_{0}\right)=\beta_{n}(n=0,1,2, \ldots)$.

Proof. We consider $N_{k}=\prod_{j \geq k} P_{w b}\left({ }^{j} E\right)$ and $u: A^{\infty}(E) \rightarrow \mathscr{S}_{w b}(E)$ defined by

$$
u(f)=\left(f\left(x_{0}\right), \hat{d} f\left(x_{0}\right), \ldots, \hat{d}^{n} f\left(x_{0}\right), \ldots\right) \in \mathscr{S}_{w b}(E),
$$

if $f \in A^{\infty}(E)$. For $k=1,2, \ldots$ fixed and $\beta=\left(\beta_{0}, \beta_{1}, \ldots, \beta_{n}, \ldots\right) \in$ $\mathscr{S}_{w b}(E)$, the function $f(x)=\sum_{j=0}^{k-1} 1 / j ! \beta_{j}\left(x-x_{0}\right)(x \in E)$ lies $A^{\infty}(E)$, $\hat{d}^{r} f\left(x_{0}\right)=\beta_{r}(0 \leq r \leq k-1)$, and $u(f)-\beta \in N_{k}$. Let \|\|$_{n, p}$ be the continuous seminorm on $A^{\infty}(E)$ defined by

$$
\|f\|=\sup _{0 \leq r \leq n} \sup _{\|x\| \leq p}\left\|\hat{d}^{r} f(x)\right\|, \quad p, n \in N, f \in A^{\infty}(E) .
$$

If $k=n+1$ and $f \in A^{\infty}(E)$ is such that $u(f) \in N_{k}$, then $D^{r} f\left(x_{0}\right)=0$ $(0 \leq r \leq n)$. On account of Theorem (1.4) the sequence $\left\{g_{\nu}\right\}_{\nu=1}^{\infty}$, where $g_{\nu}=\left(1-h_{n(\nu), \nu}\right) f_{n(\nu)}(\nu \in N)$ converges to $f$ for \|\|$_{n, p}$. Also $u\left(g_{\nu}\right)=0$ for all $\nu$. So, the hypotheses of Th. 37.3,IV, of [20] are satisfied and $u$ is surjective.

Results of the same kind of the above theorem are found in [6], in a different context.

\section{Approximation of $m$-null functions by a complex variable method. In} this section we set up certain approximation results related with the problem of knowing when $I_{m}(K)$ is equal to $\overline{J(K)}$.

We recall that a commutative Banach algebra $A$, normed by \|\| , is radical when all its elements are quasinilpotent, i.e. $\lim _{n \rightarrow \infty}\left\|a^{n}\right\|^{1 / n}=0$ for every $a \in A$; or, equivalently, $A$ has not non-zero homomorphisms. In all of $\S 3, E$ is a separable Hilbert space with $\left\{e_{n}\right\}_{n=1}^{\infty},\left\{P_{n}\right\}_{n=1}^{\infty}$ as above, 
and $K$ denotes a weakly compact subset of $\Omega$. We put $I_{p}=I_{p}(K)$ $(p=0,1, \ldots, m), J_{m}=\overline{J(K)}$, in $A^{m}(\Omega)$.

Observation. For every $p=0,1, \ldots, m$, the quotient algebra $I_{p} / J_{m}$ is radical. Indeed, for each ideal $L$ in $A^{m}(\Omega)$, if $\varphi$ is a non-zero homomorphism on $L$, there exists a unique extension $\tilde{\varphi}$ on $A^{m}(\Omega)$ of $\varphi$ defined by $\tilde{\varphi}(f)=\varphi(g)^{-1} \varphi(f g)$, for $f \in A^{m}(\Omega)$ and $g \in L$ such that $\varphi(g) \neq 0$. Now, a homomorphism $\varphi$ on $I_{p} / J_{m}$ is identified to a homomorphism on $I_{p}$, null on $J_{m}$. If $\tilde{\varphi}$ is its extension to $A^{m}(\Omega)$, we have $\tilde{\varphi}(f)=f(x)$ for all $f \in A^{m}(\Omega)$, for some $x \in \Omega$ fixed. It follows that $g(x)=0$ for every $g \in J_{m}$ and, because $A^{m}(\Omega)$ is regular, $x \in K$. Really, if $x \notin K$ and $d=\delta(x, K)-\delta(\cdot, \cdot)$ being the metric defining the weak topology on $\Omega$ ([17], p. 61), $K_{d / 2}=\{x \in \Omega: \delta(x, K) \leq d / 2\}$ is a weakly closed subset of $\Omega$, with $x \notin K_{d / 2}$. The regularity of $A^{m}(\Omega)$ implies that there exists $g \in A^{m}(\Omega)$ such that $g(x) \neq 0$ and $g\left(K_{d / 2}\right)=0$, so $g \in J_{m}, g(x) \neq 0$. Thus, $\varphi(f)=f(x)=0$ for every $f \in I_{p}$, and $I_{p} / J_{m}$ is radical.

Let $f \in A^{0}(\Omega)$ such that $f(x) \geq 0$ for every $x \in \Omega$. If $z \in C$ and $\operatorname{Re} z>0$, we define

$$
f^{z}(x)= \begin{cases}\exp (z \log f(x)), & \text { if } f(x)>0 \\ 0, & \text { if } f(x)=0\end{cases}
$$

It is immediate that $f^{z} \in A^{0}(\Omega)$ whenever $\operatorname{Re} z>0$, and it is a routine exercise, based upon the uniform continuity of each $D^{r} f(r=0,1, \ldots, m)$ and the mean value theorem, to verify that really $f^{z} \in A^{m}(\Omega)$ whenever $\operatorname{Re} z>m$ and $f \in A^{m}(\Omega)$.

LEMMA (3.1). If $f \in A^{m}(\Omega)$ and $f \geq 0$, then the map

$$
\begin{aligned}
\{z \in C: \operatorname{Re} z>m\} & \rightarrow A^{m}(\Omega) \\
z & \mapsto f^{z}
\end{aligned}
$$

is analytic.

Proof. (1) The map is continuous. If $\varepsilon>0$ and $z_{0} \in \Omega$ are given we choose $\alpha, \delta_{1}>0$ such that $\operatorname{Re} z_{0}>\alpha$ and $\operatorname{Re} z>\alpha$ if $\left|z-z_{0}\right|<\delta_{1}$. There exists $n \in N$ which verifies $2 \exp (-\alpha n)<\varepsilon$ and so

$$
\begin{aligned}
& \left|f^{z}(x)-f^{z_{0}}(x)\right| \\
& \quad \leq \exp [\operatorname{Re}(z \log f(x))]+\exp \left[\operatorname{Re}\left(z_{0} \log f(x)\right)\right]<2 \exp (-\alpha n),
\end{aligned}
$$


whenever $\log f(x)<-n$. If $\log f(x)>-n$, then $|\log f(x)|<C$ for every $x \in \Omega$ and some constant $C$. Then we choose a suitable $\delta_{2}>0$ to have

$$
\left|\exp \left[\left(z-z_{0}\right) \log f(x)\right]-1\right|<\left(\sup _{x \in \Omega}\left|f^{z_{0}}(x)\right|\right)^{-1} \varepsilon .
$$

It is clear that if $\delta=\inf \left\{\delta_{1}, \delta_{2}\right\}$ and $\left|z-z_{0}\right|<\delta$, we obtain

$$
\left|f^{z}(x)-f^{z_{0}}(x)\right|<\varepsilon .
$$

For the derivatives the discussion is analogous.

(2) The map is analytic. It is enough to apply the Morera's theorem for the triangle, in Banach-valued functions case.

We are going to see below the main result of this section. We shall use the Ahlfors-Heins theorem ([5], p. 116).

TheOREM (3.2). If $f \in I_{p},(p=0,1, \ldots, m)$ and $f \geq 0$, then $f^{m+\varepsilon} \in J_{m}$, for all $\varepsilon>0$.

Proof. It suffices to prove the theorem for $I_{0}$. Let $\Lambda$ be a continuous linear form on $I_{0} / J_{m}$. Let $f \in I_{0}$. The function $\varphi(z)=\Lambda\left(f^{m+\varepsilon+z}+J_{m}\right)$, $\operatorname{Re} z \geq 0$, satisfies the hypotheses of [5], p. 116. By using the fact that

$$
\begin{aligned}
\lim _{n \rightarrow \infty}|\varphi(n)|^{1 / n} & \leq \lim _{n \rightarrow \infty}\|\Lambda\|^{1 / n}\left\|f^{m+\varepsilon+n}+J_{m}\right\|^{1 / n} \\
& \leq \lim _{n \rightarrow \infty}\left\|\left(f+J_{m}\right)^{n}\right\|^{1 / n}=0 \quad \text { (since } I_{0} / J_{m} \text { is radical) }
\end{aligned}
$$

together with the Ahlfors-Heins theorem in a similar way as it is shown in [7], p. 94, we obtain $\varphi(z)=0$ for every $z$ such that $\operatorname{Re} z \geq 0$. So $\Lambda\left(f^{m+\varepsilon}+J_{m}\right)=0$, for each $\Lambda \in\left(I_{0} / J_{m}\right)^{\prime}$, whence $f^{m+\varepsilon} \in J_{m}$.

THEOREM (3.3). If $f \in I_{1}, f \geq 0$, and $K=f^{-1}(\{0\})$, then $f^{m} \in J_{m}$.

Proof. Let $t=m+\varepsilon$. According to Theorem (3.2) it suffices to prove that $\lim _{t \rightarrow m^{+}} f^{t}=f$ in $A^{m}(\Omega)$, and, for this, a close observation of the expressions of each derivative $D^{j} f^{t}(x), D^{j} f^{m}(x)(x \in \Omega ; 0 \leq j \leq m)$ tell us that it is enough to verify the uniform convergence on $x \in \Omega$ of $P(t) f^{t-m}(x) D f_{x} \otimes \cdots \otimes D f_{x}$ to $m ! D f_{x} \otimes \cdots \otimes D f_{x}$, where $P(t)=$ $t(t-1) \cdots(t-m+1)$. For this, we put

$$
\tau(t, x)=\left\|\left[m !-P(t) f^{t-m}(x)\right] D f_{x} \otimes \cdots \otimes D f_{x}\right\|,
$$

if $x \in \Omega, m<t \leq m+1$

$$
c_{1}=\|f\|_{1}, \quad c_{2}=\sup \left\{1, c_{1}\right\}, \quad c_{3}=\sup _{m<t \leq m+1}|P(t)| .
$$


If $\eta>0$ is given we take $\eta_{1}=\left(\eta /\left(m !+c_{2} c_{3}\right)\right)^{1 / m}$ and we obtain

$$
\tau(t, x) \leq\left[m !+|P(t)|\left|f^{t-m}(x)\right|\right]\left\|D f_{x}\right\|^{m} \leq\left[m !+c_{3} c_{2}\right] \eta_{1}^{m}=\eta,
$$

if $\|D f(x)\|<\eta_{1}$. Moreover, the set $S=\left\{x \in \Omega\right.$ : $\left.\|D f(x)\| \geq \eta_{1}\right\}$ is weakly compact in $\Omega$ and $f$ is never zero on $S$. So, $\mu=\inf _{x \in S} f(x)>0$ whence $|\log f(x)|<c_{4}, c_{4}$ constant, for every $x \in S$. Then, we choose $0<\delta<1$ such that $t-m<\delta$ implies $\left|f^{t-m}(x)-1\right|<\eta / 2 c_{3} c_{1}^{m}$ and, on the other hand, $|m !-P(t)|<\eta / 2 c_{1}^{m}$. It follows that

$$
\begin{aligned}
\tau(t, x) & \leq\left[|m !-P(t)|+|P(t)|\left|f^{t-m}(x)-1\right|\right]\left\|D f_{x}\right\|^{m} \\
& \leq\left[\left(\eta / 2 c_{1}^{m}\right)+c_{3}\left(\eta / 2 c_{3} c_{1}^{m}\right)\right] c_{1}^{m}=\eta, \quad \text { if } t-m<\delta \text { and } x \in S .
\end{aligned}
$$

We have deduced that $\sup _{x \in \Omega} \tau(t, x) \leq \eta$ whenever $t-m<\delta$.

We examine more closely the case where $m=1$.

Proposition (3.4). Let $m=1$. If $f \in I_{1}, f \geq 0$, then $f \in J_{1}$.

Proof. We construct $g \in I_{1}, g \geq 0$, such that $f^{-1}(\{0\})=K$. For $n \in N$, we choose a function $g_{n} \geq 0$ at least of class $C^{1}$ on $P_{n}(E)=C^{n}=$ $R^{2 n}$, and $g_{n}^{-1}(\{0\})=P_{n}(K), D g_{n}\left(P_{n}(K)\right)=0$. We put

$$
g(x)=\sum_{n=1}^{\infty}\left(1 / 2^{n}\left\|g_{n}\right\|_{1}\right)\left(g_{n} \circ P_{n}\right)(x), \quad \text { for every } x \in \Omega,
$$

where

$$
\left\|g_{n}\right\|_{1}=\sup _{y \in P_{n}(\Omega)}\left\{g_{n}(y),\left\|D g_{n}(y)\right\|\right\} .
$$

We have $g \in A^{1}(\Omega), g(K)=0$, and $D g(K)=0$. Moreover, if $x \in \Omega$ with $g(x)=0$ it implies $g_{n}\left(P_{n} x\right)=0$ for every $n \in N$. Thus, $P_{n} x \in P_{n}(K)$, and so there exists a sequence $\left\{y_{n}\right\}_{n=1}^{\infty} \subset K$ such that $P_{n} x=P_{n} y_{n}$ for every $n$. $K$ being a weak compact set, we can suppose that $\left\{y_{n}\right\}_{n=1}^{\infty}$ converges weakly to a point $y \in K$. If $\omega \in E^{\prime}$,

$$
\begin{aligned}
\omega\left(P_{n} y_{n}\right)-\omega(y) & =\omega\left(P_{n} y_{n}\right)-\omega\left(y_{n}\right)+\omega\left(y_{n}\right)-\omega(y) \\
& =\left[P_{n}^{*}(\omega)-\omega\right]\left(y_{n}\right)+\omega\left(y_{n}-y\right)
\end{aligned}
$$

converges to zero when $n$ goes to infinity, since $\left\{y_{n}\right\}_{n=1}^{\infty}$ is bounded and $\lim _{n \rightarrow \infty} P_{n}^{*}(\omega)=\omega$ in norm $\left(P_{n}^{*}\right.$ denotes the dual projection of $\left.P_{n}, \quad n \in N\right)$. We have proved that $\left\{P_{n} y_{n}\right\}_{n=1}^{\infty}$ converges weakly to y. As $\lim _{n \rightarrow \infty} P_{n} x=\lim _{n \rightarrow \infty} P_{n} y_{n}, \quad x=y \in K$. Finally, $f+g \in I_{1}$, $(f+g)^{-1}(\{0\})=K$, and, account of Theorem (3.3) $f+g \in J$, and $f \in J$. 
Corollary (3.5). If $f \in I_{1} \subset A^{1}(\Omega)$ is real valued and $f^{-1}(\{0\})=K$, then $f \in J_{1}$.

Proof. If $f$ is an in the statement, $f^{+} \in A^{1}(\Omega)$, where $f^{+}(x)=$ $\sup \{f(x), 0\}$ for $x \in \Omega$. Also, $f^{+} \in I_{1}$ and from Proposition (3.4), $f^{+} \in J_{1}$. If $f^{-}=f^{+}-f$, then $f^{-} \in A^{1}(\Omega), f^{-} \in I_{1}, f^{-} \geq 0$ and so $f^{-} \in J_{1}$, i.e., $f=$ $f^{+}-f^{-} \in J_{1}$.

REMARKS. (1) By operating as for $m=1$, we can prove that $I_{0} / J_{m}$ is a nilpotent algebra for any finite $m$, i.e., there is an integer power $p, p>m$, such that $f^{p} \in J_{m}$ for every $f \in I_{0}$. The power $p$ can be improved if, for instance, $f \in I_{m}$, but it remains greater than $m$.

(2) We note that when $m=0$, the mapping $z \mapsto f^{z}$ is, really, an analytic semigroup of $\{z \in C: \operatorname{Re} z>0\}$ into $A^{0}(\Omega)$, and, in general, for each compact Hausdorff space $\mathscr{K}$ this method gives a new proof of the verification of spectral synthesis in $C(\mathscr{K})$, space of continuous functions on $\mathscr{K}$.

Acknowledgment. I want ot thank the referee for this valuable suggestions.

\section{REFERENCES}

[1] R. M. Aron, Polynomial approximation and a question of G. E. Shilov, in "Approximation Theory and Functional Analysis", J. B. Prolla (ed.), Math. Studies vol. 35, North-Holland, Amsterdam, 1979, 1-12.

[2] R. M. Aron and J. B. Prolla, Polynomial approximation of differentiable functions on Banach spaces, J. R. Angew. Math., 313 (1980), 195-216.

[3] R. M. Aron, C. Herves and M. Valdivia, Weakly continuous mappings on Banach spaces, to appear in J. Funct. Anal.

[4] E. Beckenstein, L. Narici and Ch. Suffel, Topological Algebras, Math. Studies, vol. 24, North-Holland, Amsterdam, 1977.

[5] R. P. Boas, Entire Functions, Academic Press, New York, 1954.

[6] J. F. Colombeau, Infinite dimensional $C^{\infty}$ mappings with a given sequence of derivatives at a given point, J. Anal. Math. and Appl., 71, 1 (1979), 95-104.

[7] J. Esterle, A complex-variable proof of the Wiener tauberian theorem, Ann. Inst. Fourier, 30 (1980), 91-96.

[8] L. E. Fraenkel, Formulae for high derivatives of composite functions, Math. Proc. Cambridge Phil. Soc., 83 (1978), 159-165.

[9] I. Gelfand, D. Raikov and G. Shilov, Commutative Normed Rings, Chelsea Pub., New York, 1964.

[10] G. Glaeser, Etude de quelques algebres Tayloriennes, J. Anal. Math., 6 (1958), 1-124.

[11] C. Guerreiro, Whitney's spectral synthesis theorem in infinite dimensions, in "Approximation Theory and Functional Analysis", J. B. Prolla (ed.), Math. Studies, vol. 35, North-Holland, Amsterdam, 1979, 159-185. 
[12] A. Guichardet, Special Topics in Topological Algebras, Gordon-Breach, New York, 1968.

[13] J. Muñoz and J. Ortega, Sobre las álgebras localmente convexas, Collectanea Math. XX, facs., 2 (1969), 127-149.

[14] A. S. Nemirovskii, Polynomial approximation of functions in a Hilbert space, Functional Anal. App., 7, Issue 4 (1973), 331-332.

[15] Nissenzweig, $\omega^{*}$-Sequential convergence, Israel J. Math., 22 (1975), 266-272.

[16] G. Restrepo, An infinite dimensional version of a theorem of Bernstein, Proc. Amer. Math. Soc., 23 (1969), 193-198.

[17] W. Rudin, Functional Analysis, Mc-Graw Hill, New Delhi, 1973.

[18] H. H. Schaefer, Topological Vector Spaces, Springer-Verlag, New-York, 1971.

[19] J. C. Tougeron, Idéaux de fonctions différentiables, Ergeb. Math., Springer, Berlinn, 1972.

[20] F. Treves, Topological Vector Spaces, Distributions and Kernels, Academic Press, New York, 1967.

[21] J. C. Wells, Differentiable functions on Banach spaces with Lipschitz derivatives, J. Differential Geom., 8 (1973), 135-152.

Received May 16, 1983 and in revised form April 15, 1984. A part of the research in this article was supported by a Beca del Plan de Formación de Personal Investigator del M.E.C. (Spain), in the Université de Bordeaux I (France) during the academic year 1981-82.

Facultad de Ciencias

ZARAGOZA, SPAIN 



\section{PACIFIC JOURNAL OF MATHEMATICS EDITORS}

\author{
V. S. VARADARAJAN (Managing Editor) \\ University of California \\ Los Angeles, CA 90024 \\ Charles R. DePrima \\ California Institute of Technology \\ Pasadena, CA 91125 \\ R. FInN \\ Stanford University \\ Stanford, CA 94305
}

\section{HeRmanN FlaschKa \\ University of Arizona \\ Tucson, AZ 85721}

Ramesh A. Gangolli

University of Washington

Seattle, WA 98195

ROBION KIRBY

University of California

Berkeley, CA 94720

\section{C. MOORE}

University of California

Berkeley, CA 94720

\section{H. SAMELSON}

Stanford University

Stanford, CA 94305

HAROLD STARK

University of California, San Diego

La Jolla, CA 92093

\section{ASSOCIATE EDITORS}
R. ARENS
E. F. BECKENBACH
B. H. NeumanN
F. Wolf
K. Yoshida (1906-1982)

\section{SUPPORTING INSTITUTIONS}
CALIFORNIA INSTITUTE OF TECHNOLOGY

\author{
UNIVERSITY OF SOUTHERN CALIFORNIA \\ STANFORD UNIVERSITY \\ UNIVERSITY OF HAWAII \\ UNIVERSITY OF TOKYO \\ UNIVERSITY OF UTAH \\ WASHINGTON STATE UNIVERSITY \\ UNIVERSITY OF WASHINGTON
}
UNIVERSITY OF OREGON

\begin{abstract}
UNIVERSITY OF ARIZONA
UNIVERSITY OF BRITISH COLUMBIA

UNIVERSITY OF CALIFORNIA

MONTANA STATE UNIVERSITY

UNIVERSITY OF NEVADA, RENO

NEW MEXICO STATE UNIVERSITY

OREGON STATE UNIVERSITY
\end{abstract}

The Supporting Institutions listed above contribute to the cost of publication of this Journal, but they are not owners or publishers and have no responsibility for its content or policies.

Mathematical papers intended for publication in the Pacific Journal of Mathematics should be in typed form or offset-reproduced (not dittoed), double spaced with large margins. Please do not use built up fractions in the text of the manuscript. However, you may use them in the displayed equations. Underline Greek letters in red, German in green, and script in blue. The first paragraph must be capable of being used separately as a synopsis of the entire paper. In particular it should contain no bibliographic references. Please propose a heading for the odd numbered pages of less than 35 characters. Manuscripts, in triplicate, may be sent to any one of the editors. Please classify according to the scheme of Math. Reviews, Index to Vol. 39. Supply name and address of author to whom proofs should be sent. All other communications should be addressed to the managing editor, or Elaine Barth, University of California, Los Angeles, California 90024.

There are page-charges associated with articles appearing in the Pacific Journal of Mathematics. These charges are expected to be paid by the author's University, Government Agency or Company. If the author or authors do not have access to such Institutional support these charges are waived. Single authors will receive 50 free reprints; joint authors will receive a total of 100 free reprints. Additional copies may be obtained at cost in multiples of 50 .

The Pacific Journal of Mathematics is issued monthly as of January 1966. Regular subscription rate: $\$ 190.00$ a year (5 Vols., 10 issues). Special rate: $\$ 66.00$ a year to individual members of supporting institutions.

Subscriptions, orders for numbers issued in the last three calendar years, and changes of address should be sent to Pacific Journal of Mathematics, P.O. Box 969, Carmel Valley, CA 93924, U.S.A. Old back numbers obtainable from Kraus Periodicals Co., Route 100, Millwood, NY 10546.

The Pacific Journal of Mathematics at P.O. Box 969, Carmel Valley, CA 93924 (ISSN 0030-8730) publishes 5 volumes per year. Application to mail at Second-class postage rates is pending at Carmel Valley, California, and additional mailing offices. Postmaster: Send address changes to Pacific Journal of Mathematics, P.O. Box 969, Carmel Valley, CA 93924.

PUBLISHED BY PACIFIC JOURNAL OF MATHEMATICS, A NON-PROFIT CORPORATION

Copyright $\odot 1985$ by Pacific Journal of Mathematics 


\section{Pacific Journal of Mathematics}

Vol. 119, No. $2 \quad$ June, 1985

Mustafa Agah Akcoglu and Meira Falkowitz (Soshniak), A general local

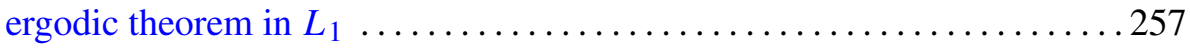

W. Wistar (William) Comfort and Lewis Chandlee Robertson,

Cardinality constraints for pseudocompact and for totally dense

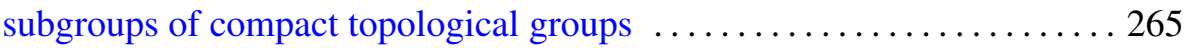

John Morse Delaurentis and Boris G. Pittel, Random permutations and

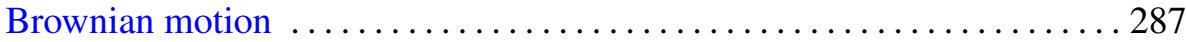

José Esteban Galé, Gel'fand theory in algebras of differentiable functions on Banach spaces ......................................... 303

Harry Gingold, On the location of zeroes of oscillatory solutions of

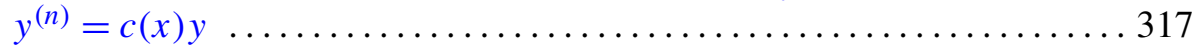

Kei Ji Izuchi, Zero sets of interpolating Blaschke products .............337

Mahesh Nerurkar, Ergodic continuous skew product actions of amenable

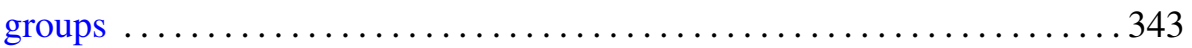

R. Owens, A maximal function characterization of a class of Hardy

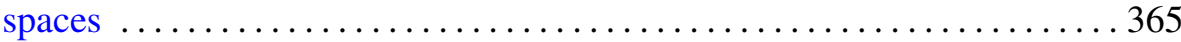

Judith Anne Packer, Point spectrum of ergodic abelian group actions and the corresponding group-measure factors $\ldots \ldots \ldots \ldots \ldots \ldots \ldots \ldots \ldots \ldots \ldots \ldots$

Judith Anne Packer, On the embedding of subalgebras corresponding to quotient actions in group-measure factors $\ldots \ldots \ldots \ldots \ldots \ldots \ldots \ldots . \ldots 4$

Iain Raeburn and Joseph L. Taylor, The bigger Brauer group and étale cohomology

David Rosen, The Diophantine equation $a x+b y=c$ in $Q(\sqrt{5})$ and other number fields

Mau-Hsiang Shih and Kok Keong Tan, Noncompact sets with convex sections

Lee Barlow Whitt, Codimension two isometric immersions between Euclidean spaces

Rodney Ian Yager, Iwasawa theory for the anticyclotomic extension 